\title{
CONSTRUÇÃO DE UM INDICADOR QUANTITATIVO (STATUS DE SAÚDE) PARA AVALIAÇÃO DA QUALIDADE DE SAÚDE ANIMAL DE PROPRIEDADES RURAIS
}

\author{
C.R. Padovani ${ }^{1}$, A.V.M. Stachissini ${ }^{2}$, H.F. da Costa ${ }^{2}$, F.F. Aragon ${ }^{1}$, J.R. Modolo ${ }^{2}$ \\ ${ }^{1}$ Universidade Estadual Paulista, Instituto de Biociências, Departamento de Bioestatística, Distrito de Rubião \\ Júnior, s/nº, CEP 18618-000, Botucatu, SP, Brasil. E-mail: jrmodolo@fmvz.unesp.br
}

RESUMO

\begin{abstract}
Visando à construção de uma ferramenta de trabalho que permitisse expressar a grandeza da saúde animal de propriedades rurais, foi construído um indicador resultante da qualidade da saúde do rebanho denominado "status de saúde animal". Como exemplo para a construção do indicador, tomaram-se os soros de 923 caprinos oriundos de 17 capris do Estado de São Paulo, nos quais se avaliaram três enfermidades e seus respectivos impactos percentuais: a artriteencefalite caprina, a neosporose e a toxoplasmose - todas de caráter infeccioso e transmissível e que ocasionam consideráveis perdas econômicas. A lógica da construção matemática do "status de saúde animal" classifica a propriedade numa variação de 0 a 100\%, conforme a positividade das enfermidades no rebanho: quanto maior a frequência acumulativa de ocorrência das doenças nos animais, menor o valor do indicador, chegando ao limite de $0 \%$ - quando todos os animais estiverem positivos às três enfermidades e de 100\% - quando todo o rebanho for negativo. Para detecção de anticorpos anti-T. gondii, anti-N. caninum e anti-CAEV, foram utilizados, respectivamente, reação de imunofluorescência indireta (RIFI $\geq 16$ ), teste de aglutinação para Neospora (NAT $\geq 25$ ) e imunodifusão em gel de agarose (IDGA positivo ou negativo). O "status de saúde animal" das propriedades rurais variou de 32,38\% a 96,40\%, de acordo com a ocorrência de positividade para as enfermidades. A característica do valor do "status de saúde animal" terá reflexo direto na cadeia de produção dentro e fora da fazenda, proporcionando não somente enormes vantagens mercadológicas, devido à expressão que a saúde animal terá para agregar valor às propriedades rurais de produção animal, mas também no consumidor final que poderá verificar a qualidade da saúde animal no início da cadeia de produção.
\end{abstract}

PALAVRAS-CHAVE: Status, saúde animal, toxoplasmose, neosporose, CAE.

\section{ABSTRACT}

BUILDING QUANTITATIVE (HEALTH STATUS) INDICATORS FOR THE ASSESSMENT OF ANIMAL HEALTH QUALITY ON FARMS. Aiming at developing a tool for assessing the magnitude of farm animal health, an indicator named herd "animal health status" was built. To illustrate the construction of the indicator, serum samples were taken from 923 goats on 17 goat farms in the state of Sao Paulo, Brazil, where three diseases were evaluated along with their impact percentages: caprine arthritis encephalitis, toxoplasmosis and neosporose - all of an infectious and transmissible nature and the cause of considerable economic losses. The mathematical rationale underlying the building of the "animal health status" indicator ranks properties on a $0-100 \%$ scale in terms of disease positivity in the herd, with the lowest indicator value indicating the highest cumulative disease frequency $(0 \%=$ all animals tested positive for the three diseases, $100 \%=$ all animals tested negative for all diseases). Anti-T. gondii, anti-N. caninum and anti-CAEV antibodies were tested using indirect immunofluorescence reaction (IIFR $\geq 16$ ), Neospora agglutination test (NAT $\geq 25$ ) and agarose gel immunodiffusion (AGID positive or negative), respectively. The animal health status of the farms ranged from $32.38 \%$ to $96.40 \%$ according to disease positivity. The animal health status value will have a direct reflection on the production chain both on and off the farm, providing not only great market advantages, due to how the expression of animal health will add value to livestock farms, as well as on the consumer who can check the quality of animal health at the beginning of the production chain.

KEY WORDS: Status, animal health, toxoplasmosis, neosporosis, CAE.

${ }^{2}$ Universidade Estadual Paulista, Faculdade de Medicina Veterinária e Zootecnia, Departamento de Higiene Veterinária e Saúde Pública, Botucatu, SP, Brasil. 


\section{INTRODUÇÃO}

Como a saúde animal é pré-requisito para a produtividadeeconômica e eficiente num sistema de produção, a ausência de enfermidades populacionais, além de ser ideal, é sempre um fator importante, já que concorre para evitar interrupção ou quebra da produtividade e consequentemente aumento de custos. A ocorrência dessas enfermidades contribui para a perda de valores agregados às propriedades com mensuráveis prejuízos ao produtor.

Atualmente, busca-se um ambiente atrativo e seguro que permita ao agronegócio atender as mudanças de hábitos dos consumidores e o aumento das exigências sanitárias brasileira e de exportação. A construção de um indicador quantitativo de "status de saúde animal", que possibilita expressar a grandeza da qualidade da saúde animal das fazendas, facilita a avaliação da saúde dos animais das propriedades rurais.

Para o desenvolvimento desseindicador desaúde, foi adotada a criação de caprinos oriundos de diferentes propriedades rurais do Estado de São Paulo a fim de avaliar a presença de três enfermidades previamente estabelecidas: toxoplasmose, neosporose e artrite-encefalite caprina.

Tais enfermidades são de caráter infeccioso, crônico e transmissível, impedindo, portanto, que os animais atinjam sua máxima performance de produção.

Os protozoários Toxoplasma gondii e Neospora caninum são responsáveis por abortos e nascimentos de crias fracas ou prematuras (LinsDaY et al., 1995; EnGEland et al., 1996; DubeY, 2003).

A infecção por $T$. gondii é relativamente comum nos pequenos ruminantes, sendo os caprinos os mais seriamente afetados. Durante a prenhez, pode causar morte embrionária, reabsorção, mumificação ou morte fetal, aborto e nascimento de natimortos ou morte perinatal. Os cabritos sobreviventes podem estar infectados, gerando prejuízos aos produtores (DubeY; AdAms, 1990; Engeland et al., 1996). As taxas de mortalidade perinatal, incluindo abortos e morte de neonatos nos rebanhos afetados, podem alcançar $50 \%$ (CHIARI et al., 1987). Nos caprinos adultos, o $T$. gondii pode também causar encefalite, nefrite, hepatite, abomasite necrosante, enterite e cistite (MEHDI et al., 1983).

Assim como a toxoplasmose, a neosporoseé uma enfermidade abortiva, causada pelo protozoário parasita Neospora caninum, estruturalmente muito similar ao T. gondii, porém distinto antigenicamente. Ele apresenta um grande número de hospedeiros, mas, até o momento, não há evidências da sua capacidade de infectar o homem (DubEY, 2003).

A infecção por $N$. caninum em ovinos e caprinos está associada a abortamentos ou nascimentos de filhotes fracos e prematuros (CORBELLINI et al., 2001). A transmissão vertical ou transplacentária ocorre originando cabritos cronicamente infectados que perpetuam a infecção na propriedade (ANDERSON et al., 1997).

A toxoplasmose e a neosporose podem ser agravadas nos casos em que os animais de produção cohabitam com gatos (Mainaretal.,1996; V An Der PuiJe et al., 2000) e cães (McAllister et al., 1998; BAsso et al., 2001; Gondim et al., 2004), respectivamente. Além do impacto econômico que a toxoplasmose pode causar, tal enfermidade desempenha um importante papel na saúde pública, uma vez que o consumo de carne eleite infectados pode predispor a transmissão zoonótica (DuBEY; BeATTIE, 1988; JITTAPALAPONG et al., 2005; CAVALCANTE et al., 2008).

A Artrite-Encefalite Caprina (CAE) é uma enfermidade de caráter persistente e curso progressivo, $\mathrm{e}$ caracteriza-se como uma síndrome multissistêmica, sendo as principais manifestações clínicas, em animais adultos, a artrite, a mastite e/ou a pneumonia, e, em caprinos jovens, a leucoencefalomielite (CORK et al., 1974).

A CAE é considerada, hoje, uma das principais doenças de caprinos, tendo alta incidência em áreas endêmicas, afetando animais de todas as idades (Modolo et al., 2003). Acarreta grandes perdas econômicas, relacionadas à baixa produção de leite, redução da performance reprodutiva, fator que resulta em um baixo aproveitamento do potencial genético dos animais infectados, além da diminuição do período de vida útil, renovação forçada do rebanho e mortalidade dos cabritos (RowE; EAST, 1997).

A partir da avaliação da presença ou ausência dessas enfermidades, aplicou-se o modelo matemático construído para quantificar o "status de saúde animal" das propriedades.

A eficiente produção de animais de interesse zootécnico, capazes de fornecer carne e leite, exige do profissional um planejamento de saúde animal, na origem da cadeia produtiva, com uma abordagem integrada sobre os fatores que interferem na de produção (Modolo et al., 2003).

Programas de saneamento de enfermidades-com a indicação do "status sanitário" e a conscientização dos produtores - poderão transformar o produto animal em fator positivo tanto para a saúde do rebanho como para a saúde pública.

Essa nova mentalidade possibilita a integração da cadeia produtiva, pondo um fim na visão segmentada da produção. Obtêm-se, dessa forma, produtos com maior aceitação no mercado, tanto no que diz respeito à valorização dos rebanhos e das barreiras comerciais como para que a indústria possa viabilizar melhor seus processos industriais com um padrão que permita atender os novos hábitos de consumo. 
O Código Zoosanitário dos Animais Terrestres da OIE (Escritório Internacional de Saúde Animal, antiga Oficina Internacional de Epizootias) instrui que seja feita a identificação dos perigos potenciais que possam ser introduzidos nas propriedades e o controle de enfermidades já instaladas (OFFICE INTERNACIONAL DES EPIZOOTIAS, 2006).

No Brasil, o Departamento de Saúde Animal do Ministério da Agricultura, Pecuária e Abastecimento (MAPA) possui um compromisso com a valorização do patrimônio pecuário nacional, mediante prevenção, controle e erradicação de algumas doenças dos animais. Dessa forma, o presente estudo visou à elaboração de um indicador quantitativo - "status de saúdeanimal" - com ointuito de expressar a grandeza da qualidade de saúde animal de propriedades em qualquer tipo de exploração de interesse zootécnico.

\section{MATERIAL E MÉTODOS}

Para exemplificar a operacionalização e a modelagem matemática do indicador, utilizaram-se animais provenientes de 17 capris, localizados em diferentes municípios do Estado de São Paulo. Foram colhidas amostras de soro de 923 animais de ambos os sexos, sem especificidade de raça, com idade acima de três meses, compondo três faixas etárias: menores que um ano, entre um e quatro anos e maiores que quatro anos. As amostras de sangue foram processadas e o soro, estocado a $-20^{\circ} \mathrm{C}$, para posterior realização de provas sorológicas: imunodifusão em gel de agarose (IDGA), para o diagnóstico da CAE; imunofluorescência indireta (RIFI), para diagnóstico da toxoplasmose; e Neospora Agglutination Test (NAT), para o diagnóstico da neosporose.

A IDGA foi realizada de acordo com Cultip et al. (1977). O antígeno foi produzido pela amostra de CAEV trazida pelo Dr. Yahia Chebloures de Laboratorie Associe de Recherches Sur Lês Lentivirus Chez Lês Petits Ruminants, INRA-ENVL, France, isolado pelo CRAWFOD et al. (1980) e replicado no interior da membrana sinovial caprina por ABREU et al. (1998). A interpretação seguiu de acordo com as regras internacionais.

Para a RIFI, os conjugados de anti-IgG específicos para espécie caprina, identificado com a fluoresceínaisotiacina, foram produzidos pelo Laboratório de Zoonoses da Faculdade de Medicina Veterinária e Zootecnia UNESP de Botucatu, SP, em conjunto com o Centro de Controle de Zoonoses, da Cidade deSão Paulo. A RIFI foi realizada segundo as descrições de CAmargo (1974) e as amostras com título $\geq 16$ foram consideradas positivas (MACHADO; LiMA, 1987).

O teste de aglutinação de Neospora foi realizado para detecção de anticorpos anti- $N$. caninum de acordo com ROMAND et al. (1998), com a linhagem
NC-1 de taquizoítos, usando o ponto de corte 1:25 determinado pelo Animal Parasitic Diseases Laboratory USDA, Beltsville, ME, USA.

Ainda durante o período de colheita de sangue, foi aplicado um inquérito para levantamento epidemiológico relacionado à toxoplasmose e à neosporose, e, também, sobre problemas reprodutivos em cada capril, conforme descrito no Quadro 1.

Quadro 1 - Inquérito realizado nas propriedades para levantamento de informações epidemiológicas e problemas reprodutivos.

\begin{tabular}{|lcc|}
\hline \multicolumn{3}{|c|}{ Características do capril } \\
\hline Presença de gatos & Sim & Não \\
Presença de cães & Sim & Não \\
Tipo de criação & Intensiva & Extensiva \\
Abortos & Sim & Não \\
“Nascimento de" natimortos & Sim & Não \\
Nascimento de cabritos debilitados & Sim & Não \\
\hline
\end{tabular}

\section{Modelagem matemática do indicador}

O procedimento matemático, utilizado para o modelo proposto de cálculo do "status de saúde do capril", pode ser empregado em qualquer outra produção animal de interesse zootécnico. Além disso, o número de enfermidades consideradas para a construção do índice é arbitrário, não sendo estipulado um mínimo ou máximo para sua elaboração. Em particular, no exemplo considerado, foram consideradas três enfermidades nos capris (CAEV, T. gondii, N. caninum).

Considerando, genericamente, que $\mathrm{R}$ enfermidades foram selecionadas para o estudo do "status" de sanidade animal, cada animal do rebanho será avaliado quanto as ocorrências (positividades) das $\mathrm{R}$ enfermidades, totalizando várias possibilidades de combinações de ocorrências na propriedade, com diferentes frequências percentuais dessas possibilidades. O número total de possibilidades consiste de diversas combinações de inúmeras respostas, ou seja, $2^{\mathrm{R}}$ agrupamentos cujos resultados apresentados podem variar desde a negatividade de todas até a presença total das enfermidades no animal. Na situação presente, o $R$ foi igual a três enfermidades $(R=$ 3), com isso têm-se oito possibilidades no rebanho: i) animal negativo para as três enfermidades; ii) animal positivo para CAEV; iii) animal positivo para $T$. gondii; iv) animal positivo para N. caninum; v) animal positivo para CAEV e T. gondii; vi) animal positivo para CAEV e N.caninum; vii) animal positivo para T. gondii e N. caninum; viii) animal positivo para as três enfermidades. 
Objetivando associar a ordem crescente de magnitude do indicador de "status" com a melhor condição de saúde do capril, foram atribuídos pesos de 1 a $\mathrm{R}+1$, conforme os agrupamentos das ocorrências de enfermidades no rebanho. Quanto maior o peso recebido pelo agrupamento, melhor a sua condição de saúde quanto à ocorrência das enfermidades e, em contrapartida, quanto menor, pior a condição.

Os agrupamentos foram constituídos levando-se em consideração o número de ocorrências das enfermidades nos animais, fato que determinou $\mathrm{R}+1$ grupos distintos. Nesse sentido, atribui-se o escore (peso) 1 ao grupo de animais que apresentavam todas as enfermidades, isto é , com as R enfermidades (situação mais desfavorável ao indicador, portanto, menor peso); o escore 2 ao grupo de animais com $(R-1)$ quaisquer enfermidades e ,assim, sucessivamente até o escore $(R+1)$ ao grupo de animais livres de enfermidades (situação mais favorável ao indicador, portanto, maior peso). No exemplo em discussão, tem: peso 1 o grupo de animais com as três enfermidades; peso 2 o grupo de animais portadores de duas: enfermidades quaisquer (CAEV e T. gondii ou CAEV e N. caninum ou T. gondii e N. caninum); peso 3 o grupo de animais portadores de uma enfermidade qualquer (CAEV ou T. gondii ou $N$. caninum) e, finalmente, peso 4 o grupo de animais negativos para todas enfermidades.

Para o cálculo numérico do indicador da condição da saúde de uma propriedade (no exemplo proposto, um capril) com um número qualquer de animais, considera-se primeiramente, o total ponderado de pontos obtidos (TP) expresso por:

$\mathrm{TP}=(\mathrm{R}+1) * \%$ animais livres de enfermidades $\left(\mathrm{P}_{0}\right)$ $+\mathrm{R} * \%$ animais com uma enfermidade $\left(\mathrm{P}_{1}\right)+(\mathrm{R}-1)$ $\%$ animais com duas quaisquer enfermidades $\left(\mathrm{P}_{2}\right)+$ $\ldots+2 * \%$ animais com $(\mathrm{R}-1)$ quaisquer enfermidades $\left(\mathrm{P}_{\mathrm{R}-1}\right)+1 * \%$ animais com as $\mathrm{R}$ enfermidades $\left(\mathrm{P}_{\mathrm{R}}\right)$, ou seja:

$\mathrm{TP}=(\mathrm{R}+1) * \mathrm{P}_{0}+\mathrm{R} * \mathrm{P}_{1}+(\mathrm{R}-1) * \mathrm{P}_{2}+\ldots+2 * \mathrm{P}$ $\mathrm{R}-1+1 * \mathrm{P}_{\mathrm{R},}$

em que TP é o total ponderado

$\mathrm{P}_{0}, \mathrm{P}_{1}, \ldots \mathrm{P}_{\mathrm{R}}$ são as porcentagens de animais de cada grupo dos capris especificadas por:

$\mathrm{P}_{0}=$ porcentagem de animais negativos (grupo de negativos);

$\mathrm{P}_{1}=$ porcentagem de animais com uma das enfermidades (grupo de positivos para uma enfermidade); $\mathrm{P}_{2}=$ porcentagem de animais com duas quaisquer enfermidades (grupo de positivos para duas enfermidades);

$P_{R}=$ porcentagem de animais com todas as enfermidades (grupo de positivos para R enfermidades).

Em continuidade, a partir do TP, estabeleceu-se o "status" de saúde animal, cujo valor SS\% consiste na padronização do TP, numa escala percentual de 0 a $100 \%$, sendo que $0 \%$ compreende a situação mais desfavorável do "status" de saúde de uma propriedade onde foram encontradas, simultaneamente, as $\mathrm{R}$ enfermidades em todos os animais, e $100 \%$ a situação mais favorável, em que tem-se uma propriedade com ausência total das enfermidades em todos os animais. Os valores intermediários foram obtidos utilizando-se a seguinte fórmula de cálculo: SS $(\%)=100$ * (TP - Pontuação Mínima)/(Pontuação Máxima - Pontuação Mínima)

e, portanto,

SS $(\%)=100 *(\mathrm{TP}-100) /(100 \mathrm{R})=(\mathrm{TP}-100) / \mathrm{R}$

Para efeito de entendimento dos cálculos dos valores de TP eSS(\%), foram utilizados como exemplo, no presente estudo, alguns capris do Estado de São Paulo, nos quais se pesquisou a ocorrência de três tipos de enfermidades $(R=3)$ de interesse econômico (A: artrite-encefalite caprina; B: neosporose; C: toxoplasmose).

Para o cálculo do "status de saúde", procedeu-se da seguinte maneira (Tabela 1):

1) Para cada capril, foram determinadas as porcentagens de animais com nenhuma, uma, duas ou três ocorrências de positividade $\left(\mathrm{P}_{0}, \mathrm{P}_{1}, \mathrm{P}_{2}\right.$ e $\left.\mathrm{P}_{3}\right)$;

$2^{\circ}$ ) Para cada grupo de ocorrência das positividades, foi estabelecido um peso de 1 a $4(R+1=4)$, sendo o valor 4 para nenhuma ocorrência de enfermidade, 3 para uma ocorrência, 2 para duas ocorrências e 1 para três ocorrências;

$3^{\circ}$ ) A partir dos pesos e as respectivas porcentagens de ocorrência no capril, determinou-se o total de pontos, dado pela fórmula:

$\mathrm{TP}=4 * \mathrm{P}_{0}+3 * \mathrm{P}_{1}+2 * \mathrm{P}_{2}+1 * \mathrm{P}_{3}$

$4^{\circ}$ ) A partir da pontuação, obteve-se a porcentagem correspondente ao "status de saúde" determinado pela seguinte fórmula:

SS $(\%)=(\mathrm{TP}-100) / 3$

5) Ovalor encontrado entre 0 e $100 \%$ indica o "status de saúde" do capril: quanto maior o valor, melhor a qualidade da saúde animal deste.

Para melhor entendimento da operacionalização, considere o capril 1 (Tabela 1 ) onde as porcentagens de ocorrência das enfermidades foram:

$\mathrm{P} 0=44,43 \%$ (nenhuma enfermidade); $\mathrm{P} 1=39,84 \%$ (uma qualquer enfermidade); P2 $=14,84 \%$ (duas quaisquer enfermidades); P3 $=0,78 \%$ (todas as três enfermidades).

Logo, $\mathrm{TP}=4 * 44,53+3 * 39,84+2 * 14,84+1$ * $0,78=328,11$ pontos.

Portanto SS $(\%)=(328,11-100) / 3=76,04 \%$.

*dados ainda não publicados. 
Tabela 1 - "Status de saúde animal" dos capris do Estado de São Paulo, de acordo com a frequência de respostas positivas às enfermidades, Botucatu.

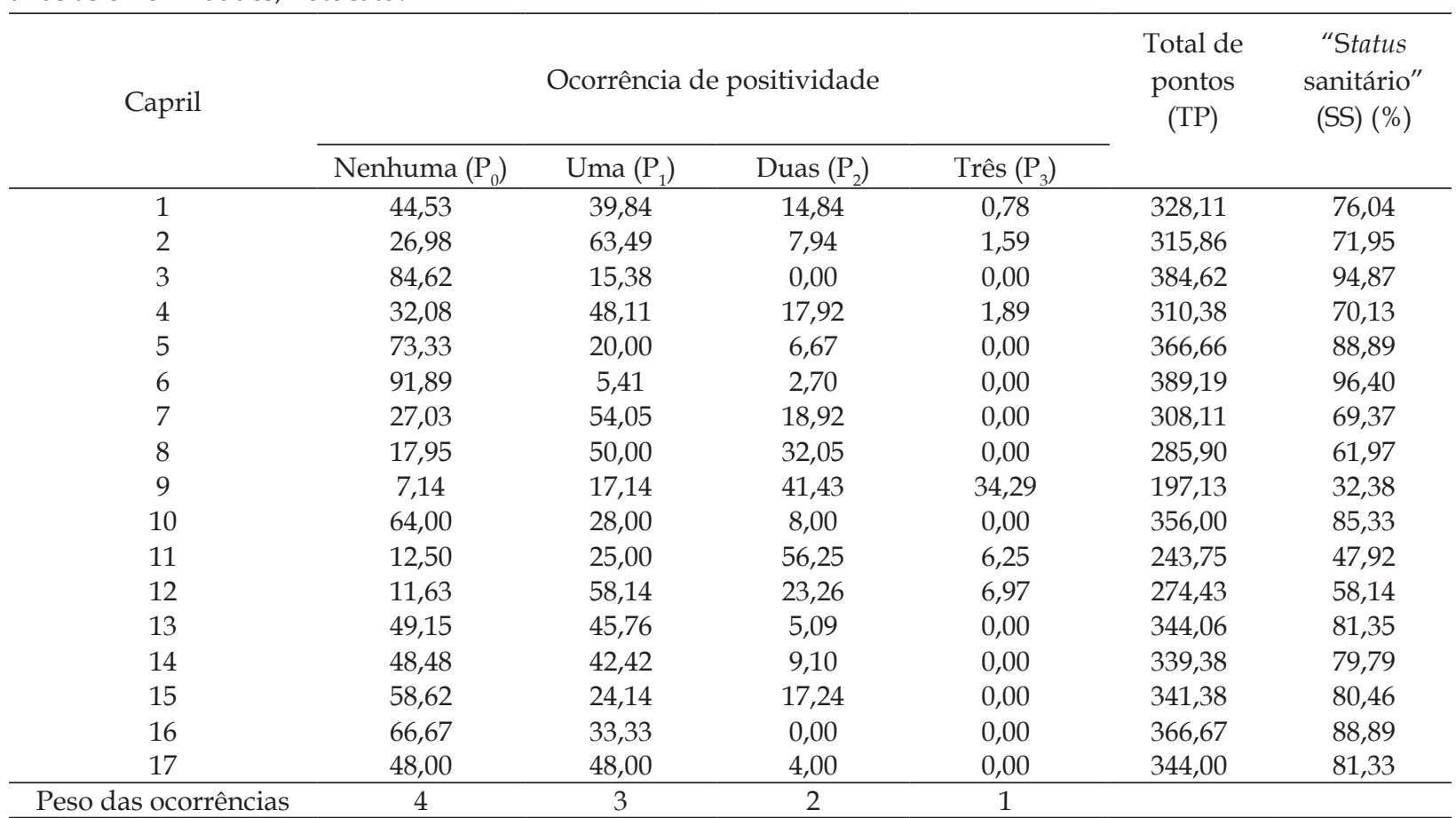

\section{RESULTADOS}

Após a avaliação das ocorrências das enfermidades, foi realizado o cálculo e chegou-se ao "status de saúde animal" atribuído para cada capril. Numa escala de 0 a $100 \%$, houve uma grande variação nos valores dos "status" entre os capris, sendo 32,38\% o menor valor atribuído àquele capril com pior condição de sanidade e $96,40 \%$ o maior valor ao capril com melhores condições de sanidade dentre os 17 capris avaliados.

A Tabela 1 ilustra o "status de saúde animal" atribuído à cada capril, de acordo com a frequência de respostas positivas ao CAEV, T. gondii e N. caninum.

\section{DISCUSSÃO E CONCLUSÃO}

As enfermidades populacionais, particularmente as de caráter crônico, são as mais importantes contribuintes para a redução permanente da produtividade, impedindo, portanto, que os animais atinjam sua máxima performance e produção econômica.

Tal impedimento pode ser agravado quando ocorre uma associação de enfermidades, descrito por STACHISSINI (2005) em um estudo o qual demonstrou que, quando houve co-infecção nos caprinos pelo vírus da CAE e pelo T. gondii ou CAE e $N$. caninum, as ocorrências de falhas reprodutivas foram significativamente maiores $(p<0,01)$. Esses dados sugerem a hipótese de que a imunossupressão causada pelo CAEV pode predispor os caprinos ao desenvolvimento de sintomas clínicos da toxoplasmose e neosporose, potencializando os riscos da ocorrência de problemas reprodutivos causados por estas enfermidades.

Atualmente, com o avanço do agronegócio e da globalização, têm ocorrido mudanças de hábitos de consumo e aumento das exigências sanitárias do mercado consumidor. Esses aspectos reafirmam não somente a necessidade de uma melhor integração da cadeia de produção da saúde animal, mas também de poder expressar a qualidade da saúde animal de propriedades rurais.

Portanto, a construção de um indicador quantitativo para expressar a grandeza da qualidade da saúde animal de propriedades de animais de produção "status de saúde animal" - poderá contribuir para avaliar a saúde dos animais na cadeia de produção dentro da porteira, refletindo, diretamente, na qualidade do produto comercializado pela propriedade rural com um impacto positivo de mercado, e na outra ponta desta cadeia, no consumidor final, podendo este identificar a qualidade da saúde animal no início do processo dentro da porteira.

Ademais, indicadores como o proposto no presente estudo poderão envolver situações mais complexas do que a apresentada na nesta pesquisa. Isto é, situações onde se envolvem gravidades das enfermidades, co-enfermidades, correlações entre as enfermidades, dentre outras. Nestas situações preserva-se a metodologia de construção do modelo. 
Porém, a complexidade biológica acarreta em complexidade matemática da função linear que expressa o "status sanitário".

Portanto, o modelo de "status de saúde animal" proposto poderá, também, ser utilizado em outros sistemas mais complexos de produção de animais de interesse zootécnico.

\section{REFERÊNCIAS}

ABREU, S.R.O.; CASTRO, R.S.; NASCIMENTO, S.A.; SOUZA, M.G. Produção de antígeno nucleoprotéico do vírus da artrite-encefalite Maedi-Visna para utilização em teste de imunodifusão em Agar gel. Pesquisa Veterinária Brasileira, v.18, p.57-60, 1998.

ANDERSON, M.L.; REYNOLDS, J.P.; ROWE, J.D. Evidence of vertical transmission of Neospora sp. Infection in dairy cattle. Journal of the American Veterinary Medical Association, v.210, p.1169-1172, 1997.

BASSO, W.; VENTURINI, L.; VENTURINI, M.C.; HILL, K.O.; SHEN, S.K.; DUBEY, J.P. First isolation of Neospora caninum from the feces of a naturally infected dog. Journal of Parasitology, v.87, p.612-618, 2001.

CAMARGO, M.E. Introdução às técnicas de imunofluo-rescência. Revista Brasileira de Patologia Clinica, v.10, p.143-169, 1974.

CAVALCANTE, A.C.R.; CARNEIRO, M.; GOUVEIA, A.M.G.; PINHEIRO, R.R.; VITOR, R.W.A. Risk factors for infection by Toxoplasma gondii in herds of goats in Ceará. Brazil. Arquivo Brasileiro de Medicina Veterinária e Zootecnia, v.60, p.36-41, 2008.

CHIARI, C.A.; LIMA, W.S.; LIMA, J.D.; ANTUNES, C.M.F. Soro-epidemiologia da Toxoplasmose caprina em Minas Gerais, Brasil. Arquivo Brasileiro de Medicina Veterinária e Zootecnia, v.39, p.587-609, 1987.

CORBELLINI, L.G.; COLODEL, E.M.; DRIEMEIR, D. Granulomatosus encephalitis in a neurologically impaired goat kid associated with degeneration of Neospora caninum tissue cysts. Journal of Veterinary Diagnostic Investigation, v.13, p.416-419, 2001.

CORK, L.C.; HADLOW, W.J.; CRAWFORD, T.B.; GORHAM, J.R.; PIPER, R.C. Infectious leukoencephalomyelitis of young goats. Journal of Infectious Diseases, v.129, p.134-141, 1974.

CRAWFORD, T.B.; ADAMS, D.S.; CHEEVERS, W.P.; CORK, L.C. Chronic arthritis in goats caused by a retrovirus. Science, v.207, p.997-999, 1980.

CULTIP, R.C.; JACKSON, T.A.; LAIRD, O.A. Immunodiffusion test for ovine progressive pneumonia. American Journal of Veterinary Research, v.38, p.1081-1084, 1977.
DUBEY, J.P. Review of Neospora caninum and neosporosis in animals. The Korean Journal of Parasitology, v.41, p.1-16, 2003.

DUBEY, J.P.; BEATTIE, C.P. Toxoplasmosis of animals and man. Boca Raton: CRC Press, 1988. 220p.

DUBEY, J.P.; ADAMS, D.S. Prevalence of Toxoplasma gondii in dairy goats from 1982 to 1984. Journal of the American Veterinary Medical Association, v.196, p.295-296, 1990.

ENGELAND, I.V.; WALDELAND, H.; KINDAHL, H.; ROPSTAD, E.; ANDERSEN, O. Effect of Toxoplasma gondii infection on the development of pregnancy and on endocrine foetal - placental function in the goat. Veterinary Parasitology, v.67, p.1-74, 1996.

GONDIM, L.F.P.; McALLISTER, M.M.; PITT, W.C.; ZEMLICKA, D.E. Coyotes (Canis latrans) are definitive hosts of Neospora caninum. International Journal for Parasitology, v.34, p.59-161, 2004.

JITTAPALAPONG, S.; SANGVARANOND, A.; PINYOPANUWAT, N.; CHIMNOI, W.; KHACHAERAM, W.; KOIZUMI, S.; MARUYAMA, S. Seroprevalence of Toxoplasma gondii infection in domestic goats in Satun Province. Thailand Veterinary Parasitology, v.127, p.17-22, 2005.

LINDSAY, D.S.; RIPPEY, N.S.; POWE, T.A.; SARTIN, E.A.; DUBEY, J.P.; BLAGBURN, B.L. Abortions, fetal death and stillbirths in pregnant pygmy goats inoculated with tachyzoites of Neospora caninum. American Journal of Veterinary Research, v.56, p.176-1180, 1995.

MACHADO, T.M.M.; LIMA, J.D. Freqüência de anticorpos anti-Toxoplasma gondii em caprinos criados sob diferentes formas de exploração no estado de Minas Gerais. Arquivo Brasileiro de Medicina Veterinária e Zootecnia, v.55, p.485-487, 1987.

MCLLISTER, M.M.; DUBEY, J.P.; LINSDAY, D.S.; JOLLEY, W.R.; WILLS, R.A.; McGRAHM, A.M. Dogs are definitive host of Neospora caninum. International Journal for Parasitology, v.28, n.9, p.1473-1478, 1998.

MAINAR, R.C.; DE LA CRUZ, C.; ASENSIO, A.; DOMINGUES, L.; VAZQUEZ-BOLAND, J.A.

Prevalence of agglutinating antibodies to Toxoplasma gondii in small ruminants of the Madri Region, Spain, and identification of factors influencing seropositivity by multivariate analysis. Veterinary Research Communications, v.20, p.153-159, 1996.

MEHDI, N.A.Q.; KAZACOS, K.R.; CARLTON, W.W. Fatal disseminated toxoplasmosis in a goat. Journal of the American Veterinary Medical Association, v.183, p.115117, 1983.

MODOLO, J.R.; STACHISSINI, A.V.M.; CASTRO, R.S.; RAVAZZOLO, A.P. Planejamento de Saúde para o Controle 
da Artrite-Encefalite Caprina. Botucatu: Cultura Acadêmica, 2003. 78p.

OFFICE INTERNATIONAL DES EPIZOOTIES. Código Zoossanitário International dos Animais Terrestres. Paris: OIE, 2006.

ROMAND, S.; THULLIEZ, P.; DUBEY, J.P. Direct agglutination test for serologic diagnose of Neospora caninum. Parasitology Resesrch, v.84, p.50-53, 1998.

ROWE, J.D.; EAST, N.E. Risk factors for transmission and methods for control of Caprine Arthritis-Encephalitis virus infection. Veterinary Clinics of North America: Food Animal Practice, v.13, p.34-53, 1997.
STACHISSINI, A.V.M. Influência da infecção pelo vírus da artrite-encefalite caprina nos perfis soro-epidemiológicos em caprinos infectados pelo Toxoplasma gondii e Neospora caninum. 2005. 103p. Tese (Doutorado) - Faculdade de Medicina Veterinária e Zootecnia, Unesp, Botucatu, 2005.

VAN DER PUIJE; W.N.A.; BOSOMPEM, K.M.; CANACOO, E.A.; WASTLING, J.M.; AKANMORI, B.D. The prevalence of anti-Toxoplasma gondii antibodies in Ghanaian sheep and goats. Acta Tropica, v.76, p.21-26, 2000.

Recebido em 12/1/10

Aceito em 22/6/11 